\title{
Use of probiotics in diets of animal or vegetable origin for broilers
}

\author{
Uso de probióticos en dietas de origen animal o vegetal para pollos \\ de engorde
}

\author{
Ricardo Nunes, ${ }^{1}$ Ph.D, Carina Scherer, ${ }^{1}$ Ph.D, Angela Poveda $\mathrm{P}_{1}{ }^{2 *}$ Ph.D, Wagner da Silva, ${ }^{1}$ \\ M.Sc, Matias Appelt, ${ }^{1}$ M.Sc, Luis Bruno, ${ }^{1}$ Ph.D. \\ ${ }^{1}$ Universidade Estadual do Oeste do Paraná, Departamento de Zootecnia. Rua Pernambuco, 1777, \\ Campus Universitário. Marechal Cândido Rondon-Paraná, Brasil. ${ }^{2}$ Universidade Estadual de Londrina, \\ Departamento de Zootecnia. Rodovia Celso Garcia Cid, PR 445 Km 380, Campus Universitário. \\ Londrina-Paraná, Brasil. *Correspondence: angelpov@gmail.com
}

Received: May 2015; Accepted: October2015.

\section{RESUMEN}

Objetivo. Evaluar el uso de probióticos en dietas con ingredientes de origen animal o vegetal sobre las variables productivas de pollos de engorde de 1 a 42 días de edad. Materiales y métodos. Fueron utilizados 1056 pollitos de un día de edad, machos de linaje Ross 308, distribuidos en un diseño experimental completamente al azar en un arreglo factorial 2X4 (dieta de origen animal o vegetal) y cuatro promotores (antibiótico, dos probióticos y un control negativo) con 6 repeticiones y 22 aves por unidad. Resultados. A los 7 días de edad hubo diferencia $(p<0.05)$ en el consumo diario de alimento, peso y ganancia de peso y a los 14 días para peso, ganancia de peso y mortalidad entre las dietas. La conversión alimenticia de 1 a 7 días tuvo interacción $(p<0.05)$ entre los tipos de dieta y los promotores. Hubo diferencia $(p<0.05)$ para el peso y la ganancia de peso a los 14 días de edad entre los promotores. A los 21 días de edad la mortalidad presentó diferencia $(p<0.05)$ para el tipo de dietas; el peso, ganancia de peso, consuno diario de alimento y conversión alimenticia presentaron diferencia para los promotores. A los 28,35 y 42 días de edad la mortalidad y el índice de eficiencia productiva presentaron diferencia $(p<0.05)$ entre el tipo de dietas. Conclusiones. El tipo de dieta y de promotor de crecimiento no afectan las variables productivas; sin embargo, la viabilidad mejora con las dietas de origen animal.

Palabras clave: Bacillus subtilis, desempeño animal, promotores del crecimiento animal (Fuente: Agrovoc).

\section{ABSTRACT}

Objective. Evaluate the use of probiotics in diets whit ingredients of animal or vegetable origin in production variables of broilers from 1 to 42 days of age. Materials and Methods. Were used 1056 one-day-old chicks, male, of lineage Ross 308, divided in to an experimental randomized design in 2X4 factorial arrangement, two origin of diet (animal or vegetable) and four promoters (antibiotic, two probiotics and a negative control) whit 6 replicates and 22 birds per unit. Results. At 7 days old there was a difference $(p<0.05)$ in daily food intake, weight and weight gain and 14 days for weight, weight gain and mortality among diets. The feed conversion of $1-7$ days did interaction $(p<0.05)$ between types of diet and promoters. There was a difference $(p<0.05)$ for weight and weight gain at 14 days of age between promoters. At 21 days of age presented mortality difference $(p<0.05)$ for the type of diets; weight, weight gain, daily feed together and feed conversion showed difference for 
developers. At 28,35 and 42 days old mortality and productive efficiency index showed difference $(p<0.05)$ between the types of diets. Conclusions. The type of diet and growth promoter did not affect production variables, however, the viability improvement animal diets.

Key words: Bacillus subtilis, animal performance, animal growth promoters (Source: Agrovoc).

\section{INTRODUCTION}

The poultry industry is constantly optimizing production to achieve better economic results and produce safer food. Including functional foods such as prebiotics in diets enhances an animal's immune system. These foods improve productive variables, act against pathogens and environmental factors like stress, act efficiently in vaccinations, and improve nutrient digestibility (1.2). They are classified by the Food and Drug Administration (FDA) of the United States as safe substances that depend on the balance of intestinal micro flora through beneficial microorganisms.

Available probiotics mainly consist of carbohydrates and are metabolized in the colon rather than the small intestine. They act as a source of energy and stimulate the growth of beneficial microorganisms and the accumulation of metabolites such as acetate, butyrate and propionate, which maintain dynamic and innate microbiota (1).

In the composition most probiotics, two bacterial genera include, Bacillus and Bifidobacterium; these genres are highlighted by having resistance to low $\mathrm{pH}$ of the stomach and tolerance to bile salts (3).

According to the Brazilian Association of Animal Recycling (ABRA), Brazil produced about 12 million tons of animal waste in 2012 and generated 3.27 million tons of meals and 1.9 million tons of fat. Animal products such as viscera, meat and bone flour are often used in broiler diets as a source of protein, and can be used to substitute soybean meal (4). The aim of this study was to evaluate the use of probiotics in diets containing animal or vegetable ingredients on the productive variables of broilers from 1 to 42 days old.

\section{MATERIALS AND METHODS}

Study site and animals: The experiment was performed at the Experimental Aviary located in the Antonio Carlos dos Santos Pessoa Experimental Station at the State University of Western Paraná, Marechal Cândido Rondon campus (Paraná-Brazil), located at an altitude of 420 meters at coordinates $24^{\circ} 33^{\prime} 21$ south

\section{INTRODUCCIÓN}

La industria avícola optimiza constantemente la producción, para alcanzar mejores resultados económicos y producir alimentos más seguros. La inclusión de alimentos funcionales como los prebióticos mejoran el sistema inmune de los animales. Estos son responsables por mejorar las variables productivas, actúan contra agentes patógenos y medio ambientales como el estrés; además, de reaccionar eficientemente a los programas de vacunación y mejorar la digestibilidad de los nutrientes $(1,2)$. Son clasificados por la Food and Drug Administration (FDA) de los Estados Unidos como sustancias seguras que depende del equilibrio de la micro flora intestinal a través de la adición de microorganismos benéficos.

Los probióticos disponibles son formados principalmente por hidratos de carbono, se caracterizan por no metabolizarse en el intestino delgado sino en el colon. Actúan como fuente de energía, estimulando el crecimiento de microorganismos benéficos, caracterizado por el acúmulo de metabolitos como acetato, butirato y propionato los cuales son responsables por mantener la microbiota dinámica e innata (1).

En la composición de la mayoría de los probióticos, dos géneros bacterianos se destacan, Bacillus y Bifidobacterium; estos géneros se destacan por tener resistencia al $\mathrm{pH}$ bajo del estómago y tolerancia a las sales biliares (3).

Según la asociación Brasileña de Reciclaje Animal (ABRA), el Brasil produjo en el año 2012 aproximadamente 12 millones de toneladas de residuos de origen animal; generando 3.27 millones de toneladas de harinas y 1.9 millones de toneladas de grasa. Los ingredientes de origen animal como las harinas de vísceras y de carne y huesos son frecuentemente utilizados en las dietas de pollos de engorde, por ser una fuente de proteína la cual puede ser incluida en sustitución a la torta de soya (4). El objetivo de este trabajo fue evaluar el uso de probióticos en dietas con ingredientes de origen animal o vegetal sobre las variables productivas de pollos de engorde de 1 a 42 días de edad. 
latitude and $54^{\circ} 03^{\prime} 25^{\prime \prime}$ west longitude, with a humid tropical pre-mountain forest climate and an average temperature of $21^{\circ} \mathrm{C}$, ranging from 16 to $27^{\circ} \mathrm{C}$ and $1700 \mathrm{~mm}$ annual rainfall.

The east-west facing experimental aviary has a concrete floor and clay tiles, and was divided into 48 boxes, $1.30 \times 1.35 \mathrm{~m}$ each. Each box had a feeder tray and a pressure bottle during the initial phase; for the other phases a semi-tubular feeder and Nipple water dispenser was used. The birds were kept warm with 250 watt infrared bulbs. The bedding material was recycled pine sawdust, covered with a centimeter of new sawdust.

Were used 1056 male broilers, day-old Ross 308. Their initial weight was $40.45 \mathrm{~g}$, from 39 week old matrices. The birds were vaccinated in the hatchery against Marek, Fowl Pox and Infectious Bronchitis. The chickens were individually weighed and randomly distributed between the experimental units.

Treatments: The experimental diets were calculated according to the feed composition and nutritional requirements proposed by Rostagno (5).

Nutritional composition and centesimal are found in table 1. Treatments are described as follows:

\section{MATERIALES Y MÉTODOS}

Sitio de estudio y animales: El experimento se realizó en el Aviario Experimental localizado en la Estación Experimental Antonio Carlos dos Santos Pessoa de la Universidad Estadual del Oeste del Paraná, campus Marechal Cândido Rondon (Paraná-Brasil) ubicada a una altitud de 420 msnm con las coordenadas entre $24^{\circ} 33^{\prime} 21^{\prime \prime}$ latitud Sur y $54^{\circ} 03^{\prime} 25^{\prime \prime}$ longitud oeste; con clima de floresta húmeda tropical pre montaña, con temperatura promedio de $21^{\circ} \mathrm{C}$, que varía de 16 a $27^{\circ} \mathrm{C}$ y precipitación anual de $1700 \mathrm{~mm}$.

El aviario experimental, de orientación esteoeste, con piso de concreto, tejas de barro, fue dividido en 48 box, con dimensiones de $1.30 \mathrm{x}$ $1.35 \mathrm{~m}$ cada, cada box poseía un comedero tipo bandeja y un bebedero de presión para la fase inicial, para las demás fases el comedero era tubular semiautomático y bebederos tipo Nipple. La calefacción de las aves fue realizada por medio de bombillos infrarrojos de 250 watts. El material utilizado para la cama fue de aserrín de pino; siendo esta su segunda utilización, tomándose cuidado en recubrir la misma con un centímetro de altura de aserrín nuevo.

Table 1. Centesimal and chemical composition of experimental diets.

\begin{tabular}{|c|c|c|c|c|c|c|c|c|}
\hline \multirow{2}{*}{ Ingredients (kg) } & \multicolumn{2}{|c|}{ Pre initial } & \multicolumn{2}{|c|}{ Initial } & \multicolumn{2}{|c|}{ Growth } & \multicolumn{2}{|c|}{ Final } \\
\hline & RA & RV & RA & RV & RA & RV & RA & RV \\
\hline Corn & 55.73 & 51.82 & 60.33 & 56.14 & 67.07 & 60.78 & 67.38 & 61.07 \\
\hline Soy cake & 35.92 & 41.22 & 30.48 & 36.02 & 22.88 & 31.19 & 21.60 & 29.92 \\
\hline Oil & 0.69 & 2.02 & 1.86 & 3.24 & 1.73 & 3.80 & 3.12 & 5.19 \\
\hline Meat and bone flour & 2.00 & -- & 2.00 & -- & 3.00 & -- & 3.00 & -- \\
\hline Intestine flour & 2.00 & -- & 2.00 & -- & 3.00 & -- & 3.00 & -- \\
\hline Calcareous 34\% & 0.96 & 1.38 & 0.86 & 1.28 & 0.40 & 1.03 & -- & 0.95 \\
\hline Bicalcium phosphate & 0.87 & 1.77 & 0.87 & 1.77 & 0.30 & 1.65 & -- & 1.49 \\
\hline Sodium chloride & 0.45 & 0.48 & 0.40 & 0.43 & 0.36 & 0.41 & 0.31 & 0.36 \\
\hline L-Lysine (78\%) & 0.31 & 0.25 & 0.25 & 0.19 & 0.31 & 0.22 & 0.29 & 0.19 \\
\hline DL-Met. (99\%) & 0.35 & 0.35 & 0.27 & 0.27 & 0.26 & 0.26 & 0.24 & 0.23 \\
\hline L-Threonine $(98 \%)$ & 0.14 & 0.13 & 0.10 & 0.08 & 0.11 & 0.08 & 0.10 & 0.07 \\
\hline Antioxidant ${ }^{1}$ & 0.02 & 0.02 & 0.02 & 0.02 & 0.02 & 0.02 & 0.02 & 0.02 \\
\hline Mineral Supplement ${ }^{2}$ & 0.05 & 0.05 & 0.05 & 0.05 & 0.05 & 0.05 & 0.05 & 0.05 \\
\hline Vitamin Supplement ${ }^{3}$ & 0.10 & 0.10 & 0.10 & 0.10 & 0.10 & 0.10 & 0.10 & 0.10 \\
\hline Choline chloride $60 \%$ & 0.06 & 0.06 & 0.06 & 0.06 & 0.06 & 0.06 & 0.06 & 0.06 \\
\hline Organic acids & 0.20 & 0.20 & 0.20 & 0.20 & 0.20 & 0.20 & 0.20 & 0.20 \\
\hline Inert & 0.15 & 0.15 & 0.15 & 0.15 & 0.15 & 0.15 & 0.15 & 0.15 \\
\hline Total & 100.00 & 100.00 & 100.00 & 100.00 & 100.00 & 100.00 & 100.00 & 100.00 \\
\hline \multicolumn{9}{|l|}{ Nutritional Composition } \\
\hline $\mathrm{EM}(\mathrm{kcal} / \mathrm{kg})$ & \multicolumn{2}{|c|}{2970} & \multicolumn{2}{|c|}{3100} & \multicolumn{2}{|c|}{3200} & \multicolumn{2}{|c|}{3300} \\
\hline Raw protein, \% & \multicolumn{2}{|c|}{23.70} & \multicolumn{2}{|c|}{21.56} & \multicolumn{2}{|c|}{19.78} & \multicolumn{2}{|c|}{19.18} \\
\hline Lysine digestible, \% & \multicolumn{2}{|c|}{1.363} & \multicolumn{2}{|c|}{1.189} & \multicolumn{2}{|c|}{1.099} & \multicolumn{2}{|c|}{1.048} \\
\hline Digestible methionine, \% & \multicolumn{2}{|c|}{0.666} & \multicolumn{2}{|c|}{0.563} & \multicolumn{2}{|c|}{0.529} & \multicolumn{2}{|c|}{0.499} \\
\hline Digestible Met+Cis, \% & \multicolumn{2}{|c|}{0.968} & \multicolumn{2}{|c|}{0.844} & \multicolumn{2}{|c|}{0.791} & \multicolumn{2}{|c|}{0.755} \\
\hline Digestible threonine, \% & \multicolumn{2}{|c|}{0.886} & \multicolumn{2}{|c|}{0.773} & & & & \\
\hline Digestible tryptophan, \% & & & & & & & & \\
\hline Digestible arginine, \% & & & & & & & & \\
\hline Calcium, \% & & & & & & & & \\
\hline Available phosphates, \% & & & & & & & & \\
\hline Sodium chloride, $\%$ & & & & & & & & \\
\hline Potassium, $\%$ & & & & & & & & \\
\hline
\end{tabular}


DAA: Animal diet with antibiotics - diet with animal products supplemented with antibiotics until 35 days.

DVA: Vegetable diet with antibiotics - diet with vegetable products and supplemented with antibiotics until 35 days.

DACN: Animal diet, negative control - diet with animal products without growth promoters.

DVCN: Vegetable diet with negative control - diet with vegetable products without growth promoters.

DAPA: Animal diet with Probiotic A - diet with animal products supplemented with Probiotic A $(0.005 \%)$ from 1 to 42 days.

DVPA: Vegetable diet with Probiotic A- diet with vegetable products supplemented with Probiotic A $(0.005 \%)$ from 1 to 42 days.

DAPB: Animal diet with Probiotic B - diet with animal products supplemented with Probiotic $A(0.04 \%)$ from 1 to 42 days.

DVPB: Vegetable diet with Probiotic B - diet with vegetable ingredients supplemented with Probiotic $A$ $(0.04 \%)$ from 1 to 42 days.

The antibiotics used in the DAA and DVA treatments were: Lasalocid 15\% (0.03\%), avilamycin $10 \%$ $(0.005 \%)$ and colisitin $8 \%(0.0094 \%)$ for the preinitial phase; lasalocid 15\% (0,06\%), avilamycin 10\% $(0.01 \%)$ and colisitin $8 \%(0.0188 \%)$ for the initial phase and narasin $10 \%(0.08 \%)$, avilamycin $10 \%$ $(0.008 \%)$ and colisitin $8 \%(0.0125 \%$ for the growth phase. Promoters were substituted in relation to inert material in the diet.

For treatments with probiotic (Bacillus subtilis), 2 products of different brands were used, one for DAPA and DVPA diets and one for DAPB and DVPB diets. Probiotics were included to replace inert material.

Sampling: At 7, 21 and 42 days all birds and feed leftovers were weighed to evaluate production parameters. Weight gain, final weight, feed intake, feed conversion and mortality were determined from 1 to 7 days, 1 to 21 days, and 1 to 42 days. Data obtained were used to calculate the index of production efficiency (IEP) at 42 days.

The maximum, minimum and current temperature and relative humidity were observed daily at $0800 \mathrm{~h}$ and $1800 \mathrm{~h}$ using two thermohygrometers installed inside the shed 5 centimeters above the birds. The lighting program was constant, with 24 hours of light (natural and artificial light).
Fueron utilizados 1056 pollos de engorde de un día de edad, machos del linaje Ross 308, con peso inicial de $40.45 \mathrm{~g}$, provenientes de matrices con 39 semanas de edad. Las aves fueron vacunadas en la incubadora contra Marek, Viruela Aviar y Bronquitis Infecciosa. Los pollos fueron pesados individualmente y distribuidos al azar entre las unidades experimentales

Tratamientos: Las dietas experimentales fueron calculadas de acuerdo a la composición de los alimentos y requerimientos nutricionales propuestos por Rostagno (5).

La composición nutricional y centesimal está descrita en la tabla 1. Los tratamientos están descritos a continuación:

DAA: Dieta Animal con Antibiótico- dieta con ingredientes de origen animal suplementada con antibiótico hasta los 35 días de edad.

DVA: Dieta Vegetal con Antibiótico- dieta con ingredientes de origen vegetal suplementada con antibiótico hasta los 35 días de edad.

DACN: Dieta Animal Control Negativo- dieta con ingredientes de origen animal sin promotor de crecimiento.

DVCN: Dieta Vegetal Control Negativo- dieta con ingredientes de origen vegetal sin promotor de crecimiento.

DAPA: Dieta Animal con Probiótico A- dieta con ingrediente de origen animal suplementada con probiótico A $(0.005 \%)$ de 1 a 42 días de edad.

DVPA: Dieta Vegetal con Probiótico A- dieta con ingrediente de origen vegetal suplementada con probiótico A (0.005\%) de 1 a 42 días de edad.

DAPB: Dieta Animal con Probiótico B- dieta con ingrediente de origen animal suplementada con probiótico A (0.04\%) de 1 a 42 días de edad.

DVPB: Dieta Vegetal con Probiótico B- dieta con ingrediente de origen vegetal suplementada con probiótico A $(0.04 \%)$ de 1 a 42 días de edad.

Los antibióticos utilizados en los tratamientos DAA y DVA fueron: Lasalocida 15\% (0.03\%), avilamicina $10 \%(0.005 \%)$ y colisitina $8 \%(0.0094 \%)$ para la fase pre inicial; lasalocida $15 \%(0,06 \%)$, avilamicina $10 \%$ $(0.01 \%)$ y colisitina $8 \%(0.0188 \%)$ para la fase inicial y narasina $10 \%(0.08 \%)$, avilamicina $10 \%(0.008 \%)$ y colisitina $8 \%(0.0125 \%$ para la fase de crecimiento. La sustitución de los promotores fue realizada en relación al material inerte de la dieta. 
Experimental Design: A completely randomized experimental design was used with a $2 \times 4$ factorial arrangement, two types of diets (animal and plant), and four different growth promoters (antibiotics, two promoters and a negative control) with 6 replicates and 22 birds per experimental unit.

The results of the production parameters were analyzed in a SAEG statistical program; joint analysis of variance and a SNK $5 \%$ test were performed.

\section{RESULTS}

From 1 to 7 days there was no interaction $(p>0.05)$ between types of diet and growth promoters for final weight, weight gain and feed intake (Table 2). Birds fed diets containing animal products had a higher $(p<0.05)$ final weight, weight gain and feed consumption from 1 to 7 days (Table 2 ).

There was interaction for feed conversion $(p<0.05)$ between the type of diet and growth promoter, where the animal diet with probiotic A showed better feed conversion (CA) when compared to the vegetable diet. Among the

Table 2. Productive variables of birds from 1 to 7 days.

\begin{tabular}{|c|c|c|c|c|c|c|}
\hline & $\mathrm{DA}$ & DCN & DPA & DPB & Mean & CV (\%) \\
\hline \multicolumn{7}{|c|}{ Initial weight (g) } \\
\hline RA & 40.44 & 40.41 & 40.47 & 40.55 & 40.47 & \multirow{3}{*}{1.63} \\
\hline RV & 40.52 & 40.36 & 40.50 & 40.37 & 40.44 & \\
\hline Mean & 40.48 & 40.37 & 40.48 & 40.44 & & \\
\hline \multicolumn{7}{|c|}{ Final weight (g) } \\
\hline RA & 188.30 & 186.30 & 185.00 & 184.10 & $185.94 a$ & \multirow[t]{3}{*}{3.55} \\
\hline RV & 187.90 & 176.60 & 179.60 & 183.10 & $181.81 \mathrm{~b}$ & \\
\hline Mean & $188.11 \mathrm{~A}$ & $181.45 B$ & $182.3 \mathrm{~B}$ & $183.6 \mathrm{~B}$ & & \\
\hline \multicolumn{7}{|c|}{ Weight gain (g) } \\
\hline RA & 147.90 & 145.90 & 144.60 & 143.60 & $145.48 a$ & \multirow[t]{3}{*}{3.09} \\
\hline RV & 147.40 & 136.20 & 139.10 & 142.70 & $141.37 b$ & \\
\hline Mean & $147.63 \mathrm{~A}$ & $141.05 B$ & $141.9 B$ & $143.15 B$ & & \\
\hline \multicolumn{7}{|c|}{ Feed consumption $(\mathrm{g})$} \\
\hline RA & 165.00 & 169.70 & 163.40 & 168.10 & $166.56 a$ & \multirow[t]{3}{*}{2.71} \\
\hline RV & 162.20 & 162.90 & 164.50 & 162.00 & $162.90 b$ & \\
\hline Mean & 163.30 & 166.30 & 163.90 & 165.00 & & \\
\hline \multicolumn{7}{|c|}{ Feed conversion* $(\mathrm{g} / \mathrm{g})$} \\
\hline RA & $0.877 \mathrm{Aa}$ & $0.911 \mathrm{Aa}$ & $0.883 \mathrm{Ab}$ & 0.909Aa & 0.896 & \multirow[t]{3}{*}{3.43} \\
\hline $\mathrm{RV}$ & $0.863 \mathrm{Ba}$ & $0.923 \mathrm{ABa}$ & $0.916 \mathrm{Aba}$ & $0.885 \mathrm{ABa}$ & 0.897 & \\
\hline Mean & 0.870 & 0.917 & 0.899 & 0.897 & & \\
\hline
\end{tabular}

DA: Diet with antibiotics; DCN: Negative control diet; DPA: Diet with probiotic A; DPB: Diet with probiotic B. *Interaction; Values followed by upper case letters in the same line differ from the SNK test (5\%); values followed by lower case letters in the same column differ from the $\mathrm{F}$ test (5\%); CV- variation coefficient.
Para los tratamientos con probiótico (Bacillus subtilis) fueron utilizados 2 productos de marcas diferentes, uno para las dietas DAPA y DVPA y otro para las dietas DAPB y DVPB. La inclusión de los probióticos fue realizada en sustitución del material inerte.

Toma de muestras: A los 7, 21 y 42 días de edad todas las aves fueron pesadas, así como las sobras del alimento, para la evaluación de los parámetros productivos. Fueron determinadas la ganancia de peso, peso final, consumo de alimento, conversión alimenticia y mortalidad en los periodos de 1 a 7 días, de 1 a 21 días y de 1 a 42 días de edad. Los datos obtenidos fueron utilizados para el cálculo del índice de eficiencia productiva (IEP), a los 42 días.

La temperatura máxima, mínima y actual, así como la humedad relativa del aire fueron observadas diariamente a las $08 \mathrm{~h} 00$ y $18 \mathrm{~h} 00$, utilizando dos termohigrómetros, instalados en el interior del galpón a una altura de 5 centímetros de las aves. El programa de iluminación utilizado fue constante, con 24 horas de luminosidad (luz natural y artificial).

Diseño Experimental: Se utilizó un diseño experimental completamente al azar con un arreglo factorial $2 \times 4$ (dos tipos de dietas (animal y vegetal) y cuatro diferentes promotores de crecimiento (antibióticos, dos promotores y un control negativo) con 6 repeticiones y 22 aves por unidad experimental.

Los resultados de los parámetros productivos fueron analizados en el programa estadístico SAEG; realizándose análisis de variancia conjunta y utilizando el test SNK 5\%.

\section{RESULTADOS}

Durante el periodo de 1 a 7 días no hubo interacción $(p>0.05)$ entre los tipos de dieta y los promotores de crecimiento para peso final, ganancia de peso y consumo de alimento (Tabla2). Las aves alimentadas con dietas que contenían ingredientes de origen animal presentan mayor $(p<0.05)$ peso final, ganancia de peso y consumo de alimento durante el período de 1 a 7 días de edad (Tabla 2).

Hubo interacción para la conversión alimenticia $(p<0.05)$ entre el tipo de dieta y el promotor de crecimiento, donde la dieta de origen animal con probiótico A presentó mejor conversión alimenticia (CA) cuando comparado con la dieta vegetal. Entre los diferentes promotores de crecimiento, el antibiótico adicionado a la dieta vegetal proporciono mejor CA $(p<0.05)$ cuando comparado con los otros tratamientos. 
different growth promoters, antibiotics added to vegetable diets provided better CA $(p<0.05)$ when compared with other treatments.

From 1-21 days no interaction ( $p>0.05)$ on productive variables between the types of diet and growth promoters was observed (Table 3 ).

Table 3. Productive values for broilers from de 1 to 21 days.

\begin{tabular}{|c|c|c|c|c|c|c|}
\hline & DA & DCN & DPA & DPB & Mean & CV (\%) \\
\hline \multicolumn{7}{|c|}{ Final weight (g) } \\
\hline RA & 974.3 & 966.9 & 957.4 & 923.0 & 955.40 & 2.83 \\
\hline RV & 969.1 & 951.1 & 955.4 & 914.9 & 947.62 & \\
\hline Mean & $971.7 \mathrm{~A}$ & $959.0 \mathrm{~A}$ & $956.4^{a}$ & 918.9B & & \\
\hline \multicolumn{7}{|c|}{ Feed consumption $(\mathrm{g})$} \\
\hline RA & 1251.2 & 1261.0 & 1240.2 & 1208.7 & 124.44 & 2.17 \\
\hline RV & 1231.7 & 1236.8 & 1243.7 & 1215.7 & 123.89 & \\
\hline Mean & $1241.4 \mathrm{AB}$ & $1248.5 \mathrm{~A}$ & 1241.9AB & $1212.2 B$ & & \\
\hline \multicolumn{7}{|c|}{ Weight gain (g) } \\
\hline RA & 933.8 & 926.5 & 917.0 & 882.5 & 914.93 & 2.97 \\
\hline RV & 928.6 & 910.7 & 914.9 & 874.5 & 907.18 & \\
\hline Mean & $931.2 \mathrm{~A}$ & $918.6 \mathrm{~A}$ & $915.9 \mathrm{~A}$ & $878.5 B$ & & \\
\hline \multicolumn{7}{|c|}{ Feed conversion $(\mathrm{g} / \mathrm{g})$} \\
\hline RA & 1.285 & 1.305 & 1.295 & 1.310 & 1.308 & 2.36 \\
\hline RV & 1.272 & 1.300 & 1.302 & 1.330 & 1.303 & \\
\hline Mean & $1.278 \mathrm{~A}$ & $1.303 \mathrm{AB}$ & $1.299 \mathrm{AB}$ & $1.320 \mathrm{~B}$ & & \\
\hline \multicolumn{7}{|c|}{ Mortality (\%) } \\
\hline RA & 5.30 & 3.03 & 3.03 & 6.82 & $4.55 a$ & 130.62 \\
\hline RV & 1.52 & 1.52 & 1.52 & 1.52 & $1.52 b$ & \\
\hline Mean & 3.41 & 2.28 & 2.28 & 4.17 & & \\
\hline
\end{tabular}

DA: Diet with antibiotics; DCN: Negative control diet; DPA: Diet with probiotic A; DPB: Diet with probiotic B. Values followed by upper case letters in the same line differ from the SNK test (5\%); CV- variation coefficient. Values followed by lower case letters in the same column differ from the $\mathrm{F}$ test $(5 \%)$; CV- variation coefficient.

Final weight, feed intake and weight gain during 1-21 days of age was lower $(p<0.05)$ and feed conversion was worse for diets containing probiotic $B$ when compared with other treatments. Mortality for this period was higher $(p<0.05)$ for birds fed animal ingredients.

From 1-42 days no interaction ( $p>0.05)$ between the type of diet and growth promoters used (Table 4) was observed. Higher mortality $(p<0.05)$ at 42 days for animals that received diets containing animal products was observed.

Viability at 42 days of age was higher $(p<0.05)$ for birds fed vegetable products, regardless of the growth promoter used. The production efficiency factor (IEP) was affected by the type of diet (Table 5).

Birds fed diets of animal products and probiotic $A$ and the negative control group had the highest $(p<0.05)$ levels of production efficiency, while
En el periodo de 1 a 21 días de edad, no se presentó interacción ( $p>0.05$ ) entre los tipos de dieta y los promotores de crecimiento sobre las variables productivas (Tabla 3 ).

El peso final, el consumo de alimento y la ganancia de peso periodo de 1 a 21 días de edad fue menor $(p<0.05)$ y la conversión alimenticia fue peor para las dietas que contenían el probiótico B cuando comparadas con los otros tratamientos. La mortalidad para este período fue mayor $(p<0.05)$ para las aves alimentadas con ingredientes de origen animal

Durante el período de 1 a 42 días no se presentó interacción ( $p>0.05)$ entre los tipos de dieta y los promotores de crecimiento utilizados (Tabla 4). Se presentó una mortalidad mayor $(p<0.05)$ a los 42 días para los animales que recibieron dietas que contenían ingredientes de origen animal.

La viabilidad a los 42 días de edad fue mayor $(p<0.05)$ para las aves alimentadas con ingredientes de origen vegetal, independiente del promotor de crecimiento utilizado. El índice de eficiencia productiva (IEP) fue influenciado por el tipo de dieta (Tabla 5).

Las aves alimentadas con dietas de origen animal y probiótico A y la dieta control negativo presentaron los mayores $(p<0.05)$ índices de

Table 4. Productive variables for broilers from 1 to 42 days.

\begin{tabular}{|c|c|c|c|c|c|c|}
\hline & DA & DCN & DPA & DPB & Mean & CV (\%) \\
\hline \multicolumn{7}{|c|}{ Final weight (g) } \\
\hline RA & 2557.1 & 2633.8 & 2617.5 & 2586.5 & 2598.72 & 3.43 \\
\hline RV & 2647.6 & 2576.7 & 2564.7 & 2656.6 & 2611.40 & \\
\hline Mean & 2602.3 & 2605.2 & 2591.1 & 2621.5 & 2605.02 & \\
\hline \multicolumn{7}{|c|}{ Feed consumption (g) } \\
\hline RA & 4520.2 & 4669.6 & 4623.2 & 4531.5 & 4586.12 & 3.05 \\
\hline RV & 4631.8 & 4598.7 & 4535.9 & 4599.1 & 4591.37 & \\
\hline Mean & 4576.0 & 4634.1 & 4579.5 & 4565.3 & 4588.72 & \\
\hline \multicolumn{7}{|c|}{ Weight gain (g) } \\
\hline RA & 2516.7 & 2593.4 & 2577.0 & 2545.9 & 2558.25 & 3.49 \\
\hline RV & 2607.1 & 2536.4 & 2524.2 & 2616.3 & 2571.00 & \\
\hline Mean & 2561.9 & 2564.9 & 2550.6 & 2581.1 & 2564.62 & \\
\hline \multicolumn{7}{|c|}{ Feed conversion $(\mathrm{g} / \mathrm{g})$} \\
\hline RA & 1.769 & 1.774 & 1.766 & 1.752 & 1.765 & 2.29 \\
\hline RV & 1.750 & 1.785 & 1.770 & 1.730 & 1.758 & \\
\hline Mean & 1.759 & 1.779 & 1.768 & 1.741 & 1.761 & \\
\hline \multicolumn{7}{|c|}{ Mortality (\%) } \\
\hline RA & 9.09 & 6.82 & 4.55 & 10.61 & 7.77a & 89.30 \\
\hline RV & 3.79 & 4.55 & 4.55 & 5.30 & $4.55 b$ & \\
\hline Mean & 6.44 & 5.68 & 4.55 & 7.95 & 6.16 & \\
\hline
\end{tabular}

DA: Diet with antibiotics; DCN: Negative control diet; DPA: Diet with probiotic A; DPB: Diet with probiotic B. Values followed by lower case letters in the same column differ from the $\mathrm{F}$ test $(5 \%)$; CV- variation coefficient. 
Table 5. Daily weight gain, viability and productive efficiency index (IEP) of broilers 1 to 42 days old.

\begin{tabular}{|c|c|c|c|c|c|c|}
\hline & DA & DCN & DPA & DPB & Mean & CV (\%) \\
\hline \multicolumn{7}{|c|}{ Daily weight gain (g) } \\
\hline RA & 59.92 & 61.75 & 61.34 & 60.62 & 60.91 & 3.49 \\
\hline RV & 62.07 & 60.39 & 60.10 & 62.30 & 61.21 & \\
\hline Mean & 60.99 & 61.07 & 60.72 & 61.46 & 61.06 & \\
\hline \multicolumn{7}{|c|}{ Viability (\%) } \\
\hline $\mathrm{RA}$ & 90.91 & 93.18 & 95.45 & 89.39 & $92.23 b$ & 5.86 \\
\hline RV & 96.21 & 95.45 & 95.45 & 94.70 & $95.45 a$ & \\
\hline Mean & 93.56 & 94.31 & 95.45 & 92.04 & 93.84 & \\
\hline \multicolumn{7}{|l|}{ IEP } \\
\hline RA & $306.6 \mathrm{Ab}$ & 322.8Aa & $330.8 \mathrm{Aa}$ & $306.3 \mathrm{Ab}$ & 316.66 & 6.88 \\
\hline RV & $340.6 \mathrm{Aa}$ & $321.7 \mathrm{Aa}$ & $323.3 \mathrm{Aa}$ & $340.5 \mathrm{Aa}$ & 331.54 & \\
\hline Mean & 323.6 & 322.2 & 327.0 & 323.4 & 324.1 & \\
\hline
\end{tabular}

DA: Diet with antibiotics; DCN: Negative control diet; DPA: Diet with Probiotic A; DPB: Diet with Probiotic B. Values followed by different uppercase letters in the same line differ among themselves in the SNK test $(5 \%)$; values followed by lower case letters in the same column differ among themselves in the $\mathrm{F}$ test $(5 \%)$; CV - variation coefficient.

diets containing animal products with antibiotics and probiotic $B$ had the lowest IEP.

\section{DISCUSSION}

Higher values obtained for final weight and weight gain for birds fed diets containing animal products may be due to the fact that these diets adequately address nutritional requirements and the nutrients are used more effectively from 1 to 7 days (6).

Improvement observed in birds supplemented with antibiotics during the first 7 days may be because these additives benefit the host, provide intestinal microbial balance, and act on the immune system (7).

The effects of probiotics on productive variables from 1 to 7 days obtained in this study were similar to those observed by Applet et al (7), who evaluated probiotics in diets with animal and vegetable products in broilers, and Bellaver et al (8), who replaced animal meal with vegetable products, and Carvalho et al (4), who evaluated animal meal, and Rigobelo et al (9) and Rocha et al (10), who evaluated probiotics in broilers.

The results for feed conversion may be due to the innumerable benefits of probiotics in the pre-initial stage, such as the best intestinal microbial balance and modulatory action (7). Improved conversion of birds fed with animal products is due to the high nutrient density and the availability of nutrients for the birds. One factor that affects production parameters of diets containing vegetable products is the content of non-starch polysaccharides which reduce eficiencia productiva, entre tanto las dietas que contenían ingredientes de origen animal con antibiótico y probiótico B presentaron los menores IEP.

\section{DISCUSIÓN}

Los mayores valores obtenidos para peso final y ganancia de peso para las aves alimentadas con dietas que contenían ingredientes de origen animal pueden ser debido a que las dietas atienden adecuadamente los requerimientos nutricionales y por la mejor utilización de los nutrientes durante el período de 1 a 7 días (6).

La mejora observada en las aves suplementadas con antibióticos durante los primeros 7 días, puede ser debido a que estos aditivos benefician al hospedero, genera un equilibrio microbiano intestinal, además, de tener acción sobre el sistema inmune (7).

Los efectos de los probióticos sobre las variables productivas de 1 a 7 días obtenidos en este trabajo fueron semejantes a los observados por Applet et al (7) que evaluaron probióticos en dietas de origen animal y vegetal en pollos de engorde, Bellaver et al (8) que sustituyeron harinas de origen animal por ingredientes de origen vegetal, Carvalho et al (4) que evaluaron harinas de origen animal, Rigobelo et al (9) y Rocha et al (10) que evaluaron probióticos en pollos de engorde.

Los resultados obtenidos para la conversión alimentar puede ser debido a los inúmeros beneficios de los probióticos en la fase pre-inicial como el mejor equilibrio microbiano intestinal y acción moduladora (7). La mejor conversión de las aves alimentadas con ingredientes de origen animal, es debida a la alta densidad nutricional y a la disponibilidad de sus nutrientes para las aves. Uno de los factores que afecta los parámetros productivos de las dietas que contienen ingredientes de origen vegetal es el contenido de polisacáridos no amiláceos los cuales reducen la digestibilidad, aumenta la viscosidad y la motilidad intestinal (11).

Resultados semejantes fueron observados por Applet et al (7) donde observaron una mejor CA en las aves alimentadas con dietas que contenían probióticos y dietas de origen animal.

Los resultados obtenidos con el probiótico $B$ sobre los parámetros productivos durante el periodo de 1 a 21 días pueden ser debido a varios factores, entre ellos por el desarrollo del tracto intestinal de las aves el cual se puede afectar por la presencia de los aditivos, además es necesario considerar 
digestibility and increase viscosity and intestinal motility (11).

Similar results were observed by Applet et al (7), who observed improved CA in birds fed diets containing probiotics and animal products.

Results obtained using probiotic $B$ on productive parameters from 1 to 21 days may be due to several factors, including the development of the intestinal tract of the birds, which can be affected by the presence of additives. Additionally, we must consider that the efficiency of probiotics depends on the qualitative and quantitative characteristics of microorganisms. The amount used may also interfere with the action of the probiotic (12). Some species, such as Bacillus subtilis, colonize with a larger number of microorganisms than, for example, Lactobacillus acidophilus, mainly due to its spore form and because it is not destroyed when manufacturing diets (13).

Similar results were obtained by Bittencurt et al (14), who evaluated the effect of probiotics on the performance of broiler chickens. The results of feed conversion were similar to those obtained by Appelt et al (7) from 1-21 days.

The absence of interaction between diets and growth promoters from 1-42 days indicates that the productive response does not depend on the use of probiotics or antibiotics (15). However, other factors that can influence the productive response are hygiene in the environment, bedding quality, health status of the animals, and the concentration and composition of microorganisms.

The lower rate of productive efficiency in animal diets with probiotic $B$ and antibiotics may be due to the high mortality that occurred with these treatments. This mortality may be associated with metabolic problems that may be related to the higher genetic potential for growth and feed efficiency of broilers. These metabolic problems usually affect birds with increased productivity and are associated with cardiovascular deficiencies and cause sudden death (16).

In conclusion, using probiotics stimulates a similar effect than that of antibiotics and a diet free of growth promoters, and can be used in broiler diets without affecting productive parameters. Diets with vegetable products are more viable in comparison with diets containing animal products. que la eficiencia de los probióticos depende de las características cualitativas y cuantitativas de los microorganismos. La cantidad utilizada también puede interferir en la acción del probiótico (12). Algunas especies como Bacillus subtilis colonizan con mayor número de microorganismos que por ejemplo Lactobacillus acidofilus debido principalmente a la forma esporulada e por no ser destruido en el proceso de fabricación de las dietas (13).

Resultados semejantes fueron obtenidos por Bittencurt et al (14) que evaluaron la influencia de los probióticos sobre el desempeño de pollos de engorde. Los resultados da conversión alimenticia fueron semejantes a los obtenidos por Appelt et al (7) durante el período de 1 a 21 días.

La ausencia de interacción de las dietas y los promotores de crecimiento durante el período de 1 a 42 días, indica que la respuesta productiva no depende de la utilización de probióticos o antibióticos (15); sin embargo, otros factores que pueden influenciar la respuesta productiva como las condiciones de higiene del ambiente, calidad de la cama, estado sanitario de los animales, concentración y composición de los microorganismos.

El menor índice de eficiencia productiva para las dietas de origen animal con el probiótico $B$ e el antibiótico, puede ser debido a la elevada mortalidad que ocurrió con estos tratamientos. Esta mortalidad puede estar asociada a problemas metabólicos los cuales pueden estar relacionados al elevado potencial genético para el crecimiento y a la eficiencia alimentar de los pollos de engorde. Estos problemas metabólicos afectan generalmente las aves de mayor productividad y están asociados a insuficiencias del sistema cardiovascular, causando la muerte súbita (16).

En conclusión el uso de los probióticos promueve un efecto semejante al de los antibióticos y a la dieta exenta de promotores de crecimiento, pudiendo ser utilizado en las dietas de pollos de engorde sin afectar los parámetros productivos. La dieta con ingredientes vegetales presenta mayor viabilidad cuando comparada con las dietas que contienen ingredientes de origen animal. 


\section{REFERENCES}

1. Ferreira $\mathrm{CL}$, Salmien $\mathrm{S}$, Grzeskowiak I, Brizuela MA; Sanchez L, Carneiro H et al. Terminology concepts of probiotic and prebiotic and their role in human and animal health. Rev Salud Anim 2011; 33(3):137-146.

2. Mokhtari R, Yazdani AR, Rezaei M, Ghorbani B. The effect of different growth promoters on performance and carcass characteristics of broiler chickens. J Anim Vet Adv 2010; 9(20); 2633-2639.

3. Barbosa FHF, Barbosa LPJL, Bambirras LHS, Aburjaile FF. Probióticos- microrganismos a favor da vida. Rev Biol Ciên Terra 2011: 11(1):11-21.

4. Carvalho CM, Fernandes EA, Carvalho AP, Caires RM, Fagundes NS. Uso de farinhas de origem animal na alimentação de frangos de corte. RPCV 2012; 111:69-73.

5. Rostagno HS, Albino LFT, Donzele JL, Gomes PC, Oliveira RF, Lopes DC et al. Tabelas brasileiras para aves e suínos: composição de alimentos e exigências nutricionais. Viçosa, MG: Universidade Federal de Viçosa, 2005.

6. Cancherini LC, Junqueira OM, Oliveira MC, Andreotti MO, Barbosa MJB. Utilização de subprodutos de origem animal em dietas formuladas com base em proteína bruta e proteína ideal para frangos de corte de 22 a 42 dias de idade. $R$ Bras Zootec 2005: 34(2):535-540.

7. Appelt MD, Nunes RV, Pozza PC, da Silva WTM, Venturi I, Nunes CGV. Níveis de probiótico de origem animal e vegetal para frangos de corte. R Bras Zootec 2010: 39(4):765-771.

8. Bellaver C, Costa CAF, Avila VS, Fraha M, Lima GJMM, Hackenhar L et al. Substituição de farinhas de origem animal por ingredientes de origem vegetal em dietas para frangos de corte. Cienc Rural 2005;35(3):671-677.
9. Rigobelo EC, Maluta RP, Ávila FA. Desempenho de frangos de corte suplementadas com probiótico. ARS Veterinaria 2011; 27(2):111-115.

10. Rocha AP, Abreu RD, Costa MCMM, Oliveira GJC, Albinati RCB, Paz AS et al. Prebióticos, ácidos orgânicos e probióticos em rações para frangos de corte. Rev Bras Saúde Prod An 2010; 11(3):793-801.

11. Silva WTM, Nunes RV, Scherer C, Pozza PC, Pozza MSS, Appelt MD. Níveis de inulina em rações de origem animal e vegetal para frangos de corte. Semina 2013; 34(6):3019-3030.

12. Kim GB, Seo YM, Kim CH, Paik IK. Effect of dietary prebiotic supplementation on the performance, intestinal microflora and immune response of broilers. Poult Sci 2011; 90:75-82.

13. Silva CR, Pinheiro LBC. Utilização de probioticos como melhoradores de desempenho em aves. Revista Eletrônica Nutritime 2008; 5(6):690-706.

14. Bittencourt LC, SILVA CC, GARCIA PDSR, DONATO DCZ, Albuquerque R, Araújo, $L F$. Influence of a probiotic on broiler performance. R Bras Zootec 2011; 40(12):2739-2743.

15. Eichner G. Alternativas na formulação de dietas vegetarianas para frangos de corte. [Tesis de Maestria]. Brasil: Universidade Federal de Rio Grande do Sul, Facultad de Agronomia; 2005.

16. Angel R. Metabolic Disorders: Limitations to growth of and mineral deposition into the broiler skeleton after hatch and potencial implications for legs problemns. J Appl Poult Res 2007; 16:138-149. 